Boise State University

ScholarWorks

2017

Data Management Plan for Graduate Identity Formation Through Teaching

Michelle Armstrong

Boise State University

Megan E. Frary

Boise State University

Donna Llewellyn

Boise State University

Paul J. Simmonds

Boise State University

Julianne Wenner

Clemson University

See next page for additional authors 
Authors

Michelle Armstrong, Megan E. Frary, Donna Llewellyn, Paul J. Simmonds, Julianne Wenner, and Albertsons Library Research Data Management Team 


\section{Data Management Plan}

\section{Types of Data Generated by the Project}

Several types of data will be produced, gathered, and analyzed:

- Demographic and census type data: All participants will be tracked in an excel database that includes their gender, ethnicity, and academic department. For graduate students, we will also include information on if they were the first in their family to obtain an undergraduate degree; and for faculty, we will include their academic rank.

- Survey data: Surveys of all of the graduate student participants will be carried out at the start of their participation, at the end of their semester of participation, and then once a year during the grant period. Our survey will be based on the survey reported on by Chermers, et. al. (2011) in the Journal of Social Issues, 67(3). In addition, all faculty involved in the project (disciplinary course instructors and graduate student advisors) will be surveyed at the start of their participation, at the end of their first semester of participation, and once a year for the remainder of the grant period. And, the teacher candidates who are partnered with the graduate student participants will also be surveyed during their term of participation.

- Interview data: A sample of each of the key populations will be chosen for interviews to probe deeper into topics surfaced from the survey data.

- Course artifacts: Artifacts such as course assignments, reflections, lesson plans, and videos of working groups and presentations from the GCOLL course will be collected and analyzed.

\section{Data and Metadata Standards}

Student demographic data and institutional data will be provided by the Institutional Research (IR), faculty data will be provided by the academic units. Data will be made available primarily in Excel formats and from the student data warehouse. Student and faculty survey data will be gathered using Qualtrics or Google Forms and will then be exported to Excel and Word for further analysis. Data from student and faculty interviews will be collected via pen and paper or in-person notetaking and transcribed into Word documents or Excel files. For interviews that warrant the data be gathered via audio recording, the audio files will be transcribed into Word documents. Project artifacts will take a variety of forms and will be documented in Word or Excel files (as appropriate, other formats such as PowerPoint, etc. might be utilized as well). All data collection activities involving human subjects will be dependent upon IRB approval and will comply with relevant guidelines established by the Office of Research Compliance.

Study-level documentation, created using the Boise State University Study-Level Documentation README.TXT Template, will include at a minimum: description of the data, structure of data files, and information on confidentiality, access policies, or other conditions of use, and data collection methods used. When appropriate, documentation will also include quality assurance procedures carried out, changes to data collection processes made over time, and related publications or presentations. Where appropriate, additional codebooks and variable-level metadata will be created and stored with the related files.

\section{Policies for Access, Sharing, and Re-Use}

Information collected during the grant project will include personally identifiable data. Additionally, the specific nature of the project will make it difficult to maintain faculty and student anonymity if the data were publicly shared. Therefore, access to all data files and related research materials will be limited to identified project staff.

Any researcher wishing to access or use the project data, whether based at Boise State or external to the university, will be required to submit to the PIs a permission request via email or printed letter. 
Researchers will need to note which files they would like to use, how they would like to use them, and if there will be any derivative materials created or published works generated based on the data.

Researchers granted access will be required to comply with any confidentiality conditions or other assurances provided during the original IRB application.

De-identified research data will be more openly accessible by request to the PIs. In addition, the research team will be preparing presentations and publications based on this project for open dissemination. One of the deliverables from the project will be a virtual Guidebook which will contain all of the lessons learned, templates, syllabi, and assignments.

\section{Data Storage and Management}

Access will be managed by the PI in accordance with the guidelines and process detailed in the Policies for Access, Sharing, and Re-use section. If the PI is unable to continue to manage access to the materials, the PI will work with SCDM and OIT to create a long-term archiving plan that is consistent with this data management plan.

In compliance with Boise State University's Data Classification Standard and Boise State University Data Use Guidelines, all files will be stored in a shared Boise State Google Drive folder. Google Drive is a licensed, private instance of the Google service which provides unlimited cloud-based storage with managed back-ups. Access will be overseen by Megan Frary and limited to PIs and authorized project personnel.

\section{Archiving and Preservation of Access}

Data used to support any published research will be reviewed for possible anonymization and archiving through ScholarWorks. Since ScholarWorks also serves as a repository for Boise State's published research articles, library staff will also create links between the archived data sets and published journal articles, conference presentations, and other related scholarly works.

In compliance with the Boise State's Office of Research Compliance IRB policy, all data and related research materials will be retained for three years past the completion of the grant. Codebooks and metadata will be collected and stored with the data to ensure long-term usability. At the end of the post-grant three year period, the PIs will work with members of the Scholarly Communications and Data Management Group to determine if further archiving is needed and if it is possible to anonymize and make the data publicly available at that point. If it is determined that the data can be publicly shared, the data and research material files will be converted into non-proprietary formats and archived in an appropriate, trusted repository. Once made publicly accessible, an associated metadata record will be created in ScholarWorks, Boise State's institutional repository, which will help facilitate discovery and citation of the materials." 